\title{
Seasonal evaluation of heat pump system for building heating and ventilation - a case study
}

\author{
Andrzej Bugaj1,", and Maciej Miniewicz ${ }^{1}$ \\ ${ }^{1}$ Wroclaw University of Science and Technology, Faculty of Environmental Engineering, \\ ul. Norwida 4/6, 50-373 Wroclaw, Poland
}

\begin{abstract}
This paper presents results obtained from seasonal evaluation of ground source heat pump (GSHP) and exhaust air heat pump (EAHP) systems used for heating and ventilation of a school building. A Matlab simulation program was developed with models of the following elements: the building thermal performance, central heating and ventilation installations, the ground source heat pump with the ground source heat exchanger and exhaust air heat pumps in air handling units. The system based exclusively on the GSHP attaining all heating needs of the central heating and ventilation installations was compared with the combined system of the GSHP and the EAHP. The analysis was based on hourly calculations of all energy capacities and COPs as well as seasonal performance factors. In addition, the energy ratings in terms of seasonal usage of delivered, renewable, auxiliary and primary energy were performed. Those energy ratings enabled the estimation of seasonal $\mathrm{CO}_{2}$ emissions in all analysed systems. The combined application of the GSHP and the EAHP in the building gave the lowest values of primary energy consumption and $\mathrm{CO}_{2}$ emissions among all considered systems.
\end{abstract}

\section{Introduction}

The main objective of reducing energy costs in heating, ventilation and air conditioning installations can be achieved by the application of energy efficient heat pumps that exclusively cover all of the heating needs of the building. Among different types of heat pumps, the ground source heat pump (GSHP) has gained quite considerable popularity in Poland and Central Europe. The GSHP system is considered to be an energy efficient alternative to conventional methods of heating and cooling buildings that is illustrated in literature [1-3]. Furthermore, the aspect of $\mathrm{CO}_{2}$ emissions reduction is highlighted as an important feature of environmental impact of a heat generation process in GSHP systems [4, 5]. Most applications of ground source heat pumps have been realized in small residential buildings. Hence, a number of theoretical and experimental studies of those GSHP system applications are well documented in literature [6-8]. One can observe that the technical, economic and ecological characteristics of those small installations are not always well applied to medium and large buildings, for example, school buildings. Some distinguished examples of large GSHP systems can also be found in the following publications [9-11].

*Corresponding author: andrzej.bugaj@pwr.edu.pl 
Those literature references show that the large size applications of GSHP systems can give quite promising results, as renewable energy installations can be the only heating source for a building without any need for auxiliary heating.

The aim of this study is to investigate the energy saving potential of using GSHP systems in medium size buildings with the design heating load (central heating and ventilation) bigger than $500 \mathrm{~kW}$. In addition, the issue of enhancing the beneficial effect of the GSHP application by introducing another heat pump type with even better performance characteristics than the GSHP is considered. The heat pump type that could compete with the GSHP in terms of performance indicators is the exhaust air heat pump (EAHP) installed in air handling units of ventilation systems. There are some EAHP applications described and evaluated in the literature $[12,13]$. However, those applications are focused on small ventilation systems, while in this study we evaluate the bigger ventilation systems carrying over $30000 \mathrm{~m}^{3} / \mathrm{h}$ of air flow.

The study described in this paper concentrates on two cases of heat pump configurations in heating and ventilation systems in an actual school building located in Wroclaw, in the south western part of Poland. The system based exclusively on the GSHP attaining all heating needs of central heating and ventilation installations is called Case 1, whereas in Case 2 the GSHP delivers heat just to the central heating and the EAHP covers the heating needs of the ventilation. Both arrangements have been compared to the reference heating source (Case 0 ) that is a gas-fired boiler covering all heating needs of the building.

The analysis of all three cases includes the calculations of relevant performance indicators as different forms of COP (coefficient of performance) and SPF (seasonal performance factor). Usually in technical analysis average values of COP are used. This study proves that the values of COP can change quite considerably with heating demand variations and different heat source temperatures. Hence, it has been decided that the heat pump operation parameters, the relevant capacities and performance indicators, should be calculated on hourly basis throughout the heating season. Moreover, the performance analysis in terms of seasonal usage of delivered, renewable, auxiliary and primary energy has been carried out for the reference situation (Case 0) and for those two heat pump configurations (Case 1 and Case 2). As a consequence of energy usage estimations, likewise seasonal $\mathrm{CO}_{2}$ emissions have been calculated for all three cases. Thus, the environmental impact of each of the chosen systems has to some extent been assessed.

\section{Case study description}

The school building in this study is a two floor construction of $6100 \mathrm{~m}^{2}$ total useable area. The building incorporates areas of different functions such as primary school, kindergarten, canteen, library and sports facility. Its design includes a thermally efficient envelope. The high thermal effectiveness is attained by applying very good insulation with the U-value of $0.17 \mathrm{~W} / \mathrm{m}^{2} \mathrm{~K}$ for walls, $0.20 \mathrm{~W} / \mathrm{m}^{2} \mathrm{~K}$ for roofs and $1.1 \mathrm{~W} / \mathrm{m}^{2} \mathrm{~K}$ for windows. Additionally, elements of passive solar techniques are introduced to the architectural design. Thus, the heating and cooling needs of the building are largely reduced without compromising the thermal comfort. The effective air supply flow of $35800 \mathrm{~m}^{3} / \mathrm{h}$ has been estimated as the total value for all areas in the building. All rooms are equipped with radiator type central heating system with design supply water temperature of $50^{\circ} \mathrm{C}$. This value of the heating water temperature is a compromise between the characteristics of water heat pumps and the size of radiators. The central heating system operates 24 hours each weekday ensuring an indoor temperature of $20^{\circ} \mathrm{C}$. The pattern of the ventilation system operation is more complex and in the standard version the system works for 12 hours from Monday to Friday. This pattern includes all anticipated schedules of ventilation operation in the building's specific areas. 
At the design stage, the investor insisted on covering all heating requirements of the building by heat pump systems. Thus, applications of different heat pump types have been evaluated for the specific needs of the building. Following that evaluation, it was decided to investigate the performance of two heat pump configurations, designated as Case 1 and Case 2 , in order to choose the most energy efficient solution for the final design. Both arrangements have been compared to the reference heating source (Case 0 ) that is a gas-fired boiler covering all heating needs of the building.

Case 1 is based on the GSHP system that delivers heat to central heating installations and heating coils in air handling units (AHUs) throughout the heating season. During the cooling season the GSHP operates in a reverse mode supplying cold water to AHUs with a ground heat exchanger (GHX) serving as a heat sink for condensers. In this case GSHP units coupled with the GHX cover all heating and cooling demands of the building.
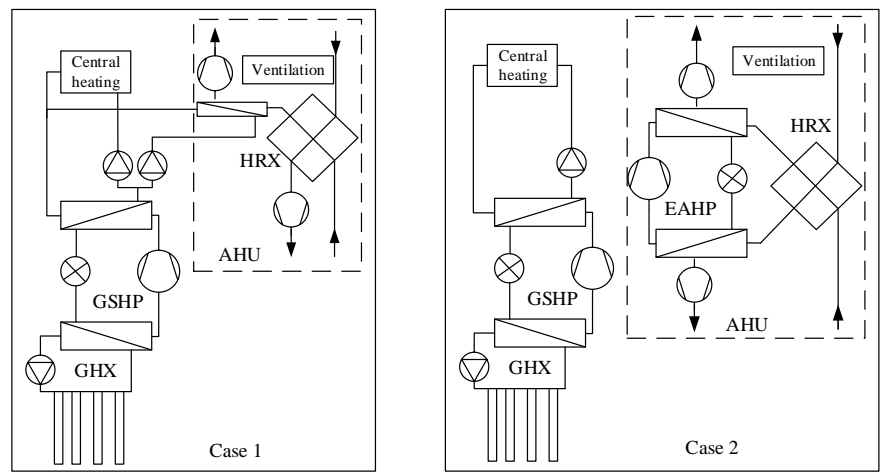

Fig. 1. The system based on the GSHP - Case 1 and the EAHP - Case 2.

Case 2 represents another configuration of heat pumps, as illustrated in Fig.1. The GSHP supplies heat just to central heating installations, whereas air handling units equipped with the exhaust air heat pump (EAHP) heat or cool ventilation air. In this case both types of heat pump, the GSHP and the EAHP, operate independently fully using their individual characteristics. In this scenario, the GSHP capacity is lower than in Case 1 because heat demand for ventilation is covered by the EAHP. Also, the ground heat exchanger (GHX), one of the most costly items of the system, can be smaller. In both cases the AHUs incorporate heat recovery plate exchangers that considerably diminish the heating demand of ventilation systems. Plate heat exchangers recover seasonally $161.03 \mathrm{MWh}$ in the overall ventilation system and rest of the load 105.33 MWh should be covered by the chosen heat pump. The cooling demand of the building ventilation system is not examined in this study.

The GSHP system uses the ground heat exchanger (GHX), which consists of vertical U-tube elements located in boreholes $120 \mathrm{~m}$ deep and with 5 meters spacing between the boreholes. The outside diameter of the pipe is $40 \mathrm{~mm}$. For the purpose of the study it has been assumed that in Case 1 and Case 2 the GHX includes 30 U-tubes and 26 U-tubes, respectively. The fluid circulating in the GHX is a glycol - water solution of $30 \%$ concentration. During the heating season the GSHP system operates continuously to cover the varying heating demand resulting from maintaining the inside temperature in the building at around $20^{\circ} \mathrm{C}$. This would be accomplished by 3 GSHP units utilizing 6 compressors. Each heat pump unit is connected with the GHX via a circulation pump providing constant brine at the flow rate recommended by the heat pump manufacturer. 


\section{System performance analyses}

Initial assessments of heat pumps performance throughout the heating season show that values of operational parameters change considerably with fluctuating heating demand and heat source temperatures. Therefore, the simulation model of the whole system has been constructed on an hour-by-hour basis. The simulation covers the following elements: the building and its heating demand for central heating and ventilation, performance of central heating and ventilation installations, the ground source heat exchanger and the heat pumps' operation.

The heating demand of the building is calculated by the straightforward hourly method recommended by the EN ISO 13790:2008 standard. This method keeps an adequate level of accuracy for the purpose of this study. The model is based on an equivalent resistance capacitance $(\mathrm{R}-\mathrm{C})$ manner. It uses an hourly time step and all building and system input data can be modified by using direct input of the hourly pattern of operation. The model makes the distinction between the internal air temperature and the internal surfaces. This approach increases the accuracy by taking into account radiative and convective parts of solar, lighting and other internal heat gains. The calculation method is based on simplifications of the heat transfer between the internal and external environments. Also, heat losses due to the infiltration process are considered. The full set of equations for this simple hourly method is given in the ISO standard documentation. The building simulation model has been run with the local climate data in an hourly pattern of the reference year. The calculation began on the 1st of October and finished on the $25^{\text {th }}$ of April, with all week days as well as daily operation schedules recognised.

The next step of the simulation procedure has been modelling of the heat pumps' performance. In both cases it is assumed that the GSHP thermal power QHP covers the heating demand, which changes hourly. The compressor power is calculated as the ratio between the $\mathrm{Q}_{\mathrm{HP}}$ and the COP. The COP depends on the brine temperature $\mathrm{T}_{\mathrm{b}}$ at the entrance of the GSHP evaporator and the supply water temperature $\mathrm{T}_{\mathrm{w}, \mathrm{s}}$ leaving the GSHP condenser. The COP formula is in the form of polynomial correlation obtained from a quadratic regression curve fitting to the catalogue data. In Case 2, the GSHP system exclusively covers the heating requirements of the central heating whereas ventilation installations are separated from the GSHP system. The heating process in the air handling units is carried out by recovery heat exchangers and exhaust air heat pumps (EAHPs). The model of the heat recovery exchanger is based on temperature efficiency values given by the manufacturer. As for the EAHP unit, it is assumed that its thermal power $\mathrm{Q}_{\mathrm{V}}$ covers the rest of the ventilation heating demand. The model of EAHP uses a similar formulation method to the GSHP model. The compressor power is calculated as the ratio between the thermal power and the COP. The COP value depends on the air supply temperature and the air temperature at the evaporator inlet. The COP formulation is taken from a quadratic regression curve fitting the data delivered by the manufacturer of the air handling units.

The simulation procedure should include also a model of the ground heat exchanger (GHX). One can find in the relevant literature a number of complex models that can predict transient heat transfer in a vertical U-tube GHX. The simplified model of GHX has been formulated in this study. In this model the borehole is assumed as an infinite cylinder surrounded by a homogenous ground of constant properties. Thus, the ground is regarded as an infinite medium with the initial uniform temperature. The thermal capacitances of the borehole as well as the axial heat flow in the grout and pipe walls are negligible as the borehole area is much smaller than the infinite ground around it. Hence, the heat transfer can be treated as a steady state one-dimensional process. This type of model can be recommended for engineering analysis. 
The performance of the heat pump unit is usually characterized by the well-known indicator the unit coefficient of performance $\mathrm{COP}_{\mathrm{u}}$ (ratio of thermal power to compressor power). Nevertheless, the system coefficient of performance, $\mathrm{COP}_{\mathrm{s}}$ gives the broader picture of heat pump systems taking into account the auxiliary energy required to run brine circulation pumps or additional fans. For the GSHP the $\mathrm{COP}_{\mathrm{s}}$ takes the form of:

$$
C O P_{s}=Q_{H} /\left(P_{c}+P_{c p}\right)
$$

where $Q_{H}$ is the heating capacity of the GSHP, $P_{c}$ the compressor power and $P_{c p}$ the circulation pumps power. The $\mathrm{COP}_{\mathrm{s}}$ for the exhaust air heat pump, the EAHP, is assumed to have the same value as COP for the unit because the combined fan power in the air handling unit with the EAHP is in a similar range as in the conventional AHU.

In order to illustrate the effect of seasonal operation of the heat pump system one can use the heating seasonal performance factor defined as the ratio of seasonal heating requirements covered by the heat pump to all energy inputs into the system. The SPF for the GSHP is calculated as follows:

$$
S P F=\int\left(Q_{H} /\left(P_{c}+P_{c p}\right)\right) d \tau
$$

where $\tau$ is time of the season.

The final analysis carried out for all three cases (Case 0, Case 1 and Case 2) was focused on the energy ratings of the described systems. The ratings have been performed according to European standard EN 15603:2008 [27], taking into account different forms of energy, such as delivered energy, primary energy, renewable energy and auxiliary energy. For the reference situation of Case 0 when all heating needs are covered by a gas-fired boiler, the delivered energy is calculated as:

$$
\begin{gathered}
E_{d e l}=E_{H V} / \eta_{t} \\
\eta_{t=} \eta_{b} \eta_{s} \eta_{d} \eta_{\mathrm{c}}
\end{gathered}
$$

where $E_{H V}$ is the energy needed for heating and ventilation, $\eta_{t}$ the total efficiency of the system taking into account boiler efficiency $\eta_{b}$, storage efficiency $\eta_{s}$, distribution efficiency $\eta_{d}$, and control efficiency $\eta_{c}$. The primary energy is calculated then as:

$$
E_{\text {prim }}=E_{\text {del }} F_{g}+E_{\text {aux }} F_{e l}
$$

where $F_{g}$ is the primary energy factor for gas heating, $F_{e l}$ the primary energy factor for electricity and $E_{\text {aux }}$ is auxiliary electrical energy. The following values of these factors have been taken for the study: $F_{g}=1.1$ and $F_{e l}=3.0$. The delivered energy for the GSHP and the EAHP is determined by the following formula:

$$
E_{d e l}=\int\left(Q_{H} / C O P\right) d \tau
$$

where $Q_{H}$ and COP is integrated throughout the heating season. The primary energy for both types of heat pumps is calculated as:

$$
E_{\text {prim }}=\left(E_{d e l, G S H P}+E_{d e l, E A H P}+E_{a u x}\right) F_{e l}
$$

In addition to the primary energy rating, a carbon dioxide rating has also been performed as stated by the standard EN 15603:2008. The combined environmental impact of all 
greenhouse gas compounds is commonly normalized to the specific effect of $\mathrm{CO}_{2}$ and all emissions are expressed in $\mathrm{CO}_{2}$ equivalents. For the purpose of this study the emissions are just expressed in the mass of $\mathrm{CO}_{2}$. Emission factors for heat production with the gas boiler, $\mathrm{EF}_{\mathrm{h}}$ and electricity production, $\mathrm{EF}_{\mathrm{el}}$, representative in the local energy production market are introduced in order to calculate actual emissions. The following values are currently used in Poland: $\mathrm{EF}_{\mathrm{h}}=0.202 \mathrm{~kg} \mathrm{CO} / \mathrm{kWh}$ and $\mathrm{EF}_{\mathrm{el}}=0.812 \mathrm{~kg} \mathrm{CO} / \mathrm{kWh}$.

\section{Results and discussion}

The building heating load was divided into two parts, the central heating part and the ventilation part, and then calculated hour-by-hour throughout the heating season from $1^{\text {st }}$ of October to $25^{\text {th }}$ of April. The simulations take into account the weather data for the reference year as well as the operational pattern of the building. The heating load profiles are constructed for heating and ventilating systems. The load profiles can give an indication of the system behaviour during operation time and help choose an optimal number of heat pump units to properly cover all of the heating needs. The central heating load profile was calculated throughout the heating season lasting about 5000 hours.

A common practice in describing the performance of heat pump system is to use an average or a reference value of COP. In this study coefficients of performance are calculated on an hour-by-hour basis throughout the heating season and then are weighted for a given time period, e.g. a day or a month. Thus, COPs values take into account the actual heating demand of the building. Case 1 represents a situation when both central heating and ventilation heating needs are covered exclusively by the GSHP system. Fig.2 shows the

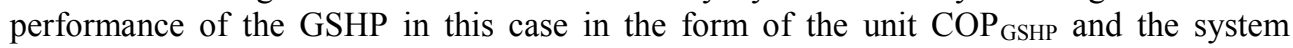
$\mathrm{COP}_{\mathrm{s}, \mathrm{GSHP}}$.

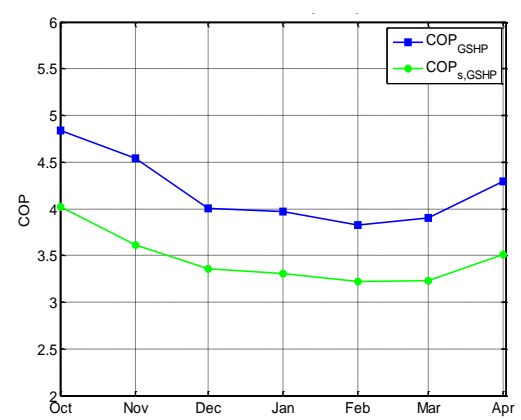

Case 1

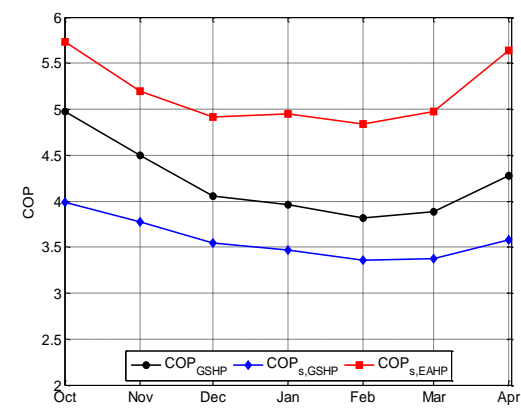

Case 2

Fig. 2. Unit and system COP values in Case 1(only GSHP) and COP values in case 2 (GSHP and EAHP together).

The COPs values are a little lower at the end of the season despite smaller heating demand than at the beginning of the season due to ground temperature degradation. However, monthly average values of $\mathrm{COP}_{\mathrm{s}}$ are quite satisfactory. There are some hours during the coldest months when values of $\mathrm{COP}_{\mathrm{s}, \mathrm{GSHP}}$ drop to a low point around 2.5 , but the monthly average values weighted with time are always above 3.0 and vary between the lowest average monthly value of 3.22 and the highest value of 4.02 with the seasonal average value of 3.46.

In Case 2 the GSHP system supplies heat to the central heating while exhaust air heat pumps (EAHPs) cover the heating needs of the ventilation air. Monthly average values of $\mathrm{COP}_{\mathrm{s}}$ in this case are depicted in Fig.2. For the EAHP the system COP $\mathrm{s}$,EAHP is on par with 
the unit COP and their values are distinctly higher than those of COP $\mathrm{s}_{\mathrm{GSHP}}$. The monthly average value of $\mathrm{COP}_{\mathrm{s}, \mathrm{EAHP}}$ ranges between 4.83 and 5.73 with the seasonal average value of 5.18. Thus, the application of the EAHP instead of the GSHP in the ventilation system seems to be quite an efficient solution.

The seasonal operation effect of the heat pump systems is illustrated by seasonal performance factors for both cases in Fig. 3 .

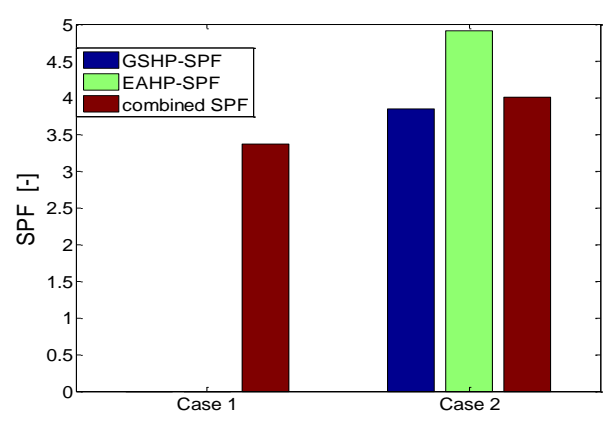

Fig. 3. SPF values for case 1 and case 2.

The SPF value for the GSHP system in Case 1 is around 3.36. In Case 2, where both heat pumps operate, the SPF for the GSHP and the EAHP are 3.84 and 4.91, respectively. The combined SPF for the case 2 reaches value of 4.0. This relatively small difference in SPF values between two cases is again caused by much shorter operation of the EAHP in comparison to the GSHP during the heating season.

The seasonal estimates give a broad picture of energy usage and enable the primary energy and $\mathrm{CO}_{2}$ ratings to be made in all three systems. The results of primary energy ratings are shown in Fig. 4.

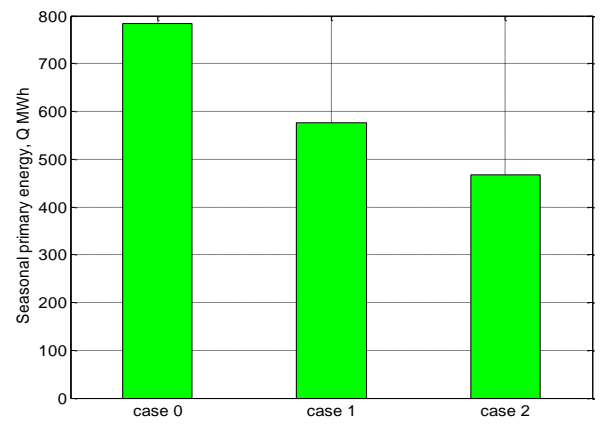

Fig. 4. Seasonal primary energy usage.

The highest primary energy usage occurs in the reference situation of Case 0 with the PEF (primary energy factor) of 1.2 for gas heating. The GSHP system in Case 1 consumes a smaller amount of primary energy despite a much bigger value of the PEF, 3.0 for electricity. And the lowest consumption of primary energy takes place in Case 2, when the GSHP and the EAHP operate together. 


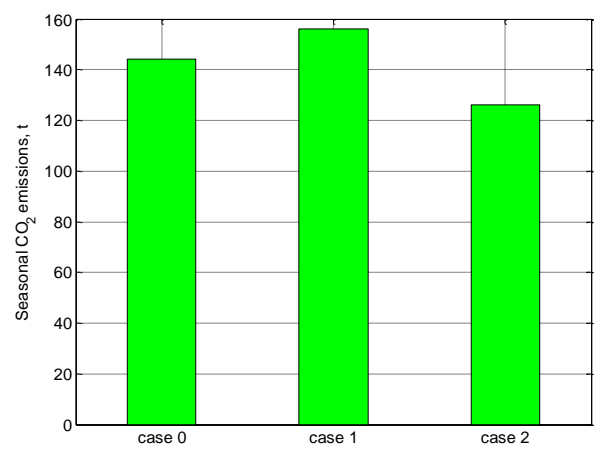

Fig. 5. Seasonal $\mathrm{CO}_{2}$ emissions for three cases.

The $\mathrm{CO} 2$ ratings performed for all three cases in accordance with the European standard EN 15603:2008 give a less favourable picture for heat pumps than that with the primary energy ratings. The seasonal $\mathrm{CO}_{2}$ emissions for the reference Case 0 with a gas-fired boiler are lower than in Case 1 with the GSHP. However, some savings in CO2 emissions occur in Case 2 when both the GSHP and the EAHP operate with the combined SPF of 4.0. The reason for this situation is the existence of the high carbon electricity mix in Poland. It is noteworthy that during recent years the changing electricity mix has reduced emissions from $1.12 \mathrm{~kg} \mathrm{CO} / \mathrm{kWh}$ to $0.812 \mathrm{~kg} \mathrm{CO} / \mathrm{kWh}$ allowing most efficient heat pumps to be an almost effective carbon saving measure. Fig. 5 illustrates the $\mathrm{CO} 2$ ratings for all three cases.

\section{Conclusions}

The presented study has focused on assessing an optimal arrangement of heat pumps that could cover all central heating and ventilation needs of a school building. The GSHP system assisting central heating and ventilation installations has been compared in terms of seasonal performance with the combined system of the GSHP and the EAHP. The analysis is based on hour-by-hour calculations of all energy capacities and COPs as well as seasonal performance factors. Additionally, energy ratings in terms of seasonal usage of delivered and primary energy have been performed. These energy ratings have enabled an estimate of seasonal $\mathrm{CO}_{2}$ emissions of all analysed systems.

The study shows that the GSHP system can fully support the building central heating and ventilation installations with the system $\mathrm{COP}_{\mathrm{s}, \mathrm{GSHP}}$ varying between the lowest monthly value of 3.22 for February and the highest value of 4.02 for October. The introduction of exhaust air heat pumps (EAHPs) to the ventilation system has increased the beneficial effect of GSHP application. The $\mathrm{COP}_{\mathrm{s}, \mathrm{EAHP}}$ is considerably higher than the $\mathrm{COP}_{\mathrm{s}, \mathrm{GSHP}}$ with the monthly average value of $\mathrm{COP}_{\mathrm{s}, \mathrm{EAHP}}$ varying between 4.83 for February and 5.73 for October. In addition, the SPF for the EAHP is substantially bigger than that for the GSHP. SPF values for the GSHP and the EAHP are 3.84 and 4.91, respectively. The combined SPF for simultaneous usage of the two heat pumps gives a value of 4.0 and it is close to the SPF for just the GSHP due to the specific pattern of ventilation operation. In the case of longer operation of the EAHP the combined SPF can extend to higher values.

The seasonal estimates have given quite an extensive view of energy usage and allowed us to make primary energy and $\mathrm{CO}_{2}$ ratings in the described systems. The GSHP system in Case 1 consumes less primary energy than the gas heating in Case 0 and the combined system of the GSHP and the EAHP in Case 2 uses a smaller amount of primary energy than the unaccompanied GSHP in Case 1. The $\mathrm{CO}_{2}$ ratings give a less favourable picture than that of 
primary energy ratings. The seasonal $\mathrm{CO}_{2}$ emissions in Case 0 with a gas-fired boiler are lower than in Case 1 (only the GSHP) but the lowest CO2 emissions are produced in Case 2 with the combined operation of the GSHP and the EAHP.

\section{References}

1. K. J. Chua, S. K. Chua, W. M. Yang, Appl. Energy 87, 3611-3624 (2010)

2. J. S. Self, B. V. Reddy, M. A. Rosen, Appl. Energy 101, 341-348 (2013)

3. Y. Man, Y. Yang, J. Wang, Appl. Energy 97, 913-920 (2012)

4. P. Blum, G. Campillo, W. Munch, T. Kolbel, Renew. Energy 35, 122-127 (2010)

5. P. Bayer, D. Saner, S. Bolay, Renew. Sustain. Energy Rev. 16, 1256-1267 (2012)

6. P. J. Boait, D. Fan, A. Strafford, Energy and Buildings 43, 1968-1976 (2011)

7. A. Strafford, D. Lilley, Energy and Buildings 49, 536-543 (2012)

8. A. S. Safa, A. S. Fung, R. Kumar, Renew. Energy 83, 565-575 (2015)

9. E. Kim, J. Lee, Y. Jeong, Energy and Buildings 50, 1-6 (2012)

10. X. Q. Zhai, X. L. Wang, H. T. Pei, Y. Yang, Appl. Thermal Eng. 48, 105-116 (2012)

11. M. A. Rosen, S. Koohi-Fayegh, Geothermal Energy (Wiley, 2017)

12. S. Riffat, M. Gilott, Appl. Thermal Eng. 22, 839-845 (2002)

13. K. B. Mercer, J. E. Braun, ASHRAE Trans. 111, 890-900 (2005) 